\title{
Exact models for Hall current reconnection with axial guide fields
}

\author{
I. J. D. Craig ${ }^{\text {a) }}$ \\ Department of Mathematics, The University of Waikato, Private Bag 3105, Hamilton, New Zealand \\ P. G. Watson ${ }^{\text {b) }}$ \\ Center for Magnetic Reconnection Studies, Institute for Fusion Studies, The University of Texas at Austin, \\ Austin, Texas 78712
}

(Received 28 June 2004; accepted 29 September 2004; published online 6 December 2004)

\begin{abstract}
This paper employs an analytic reconnection model to investigate the conditions under which Hall currents can influence reconnection and Ohmic dissipation rates. It is first noted that time dependent magnetohydrodynamic systems can be analyzed by decomposing the magnetic and velocity fields into guide field and reconnecting field components. A formally exact solution shows that Hall currents can speed up or slow down the reconnection rate depending on the strength and orientation of the axial guide field. In particular, merging solutions are developed in which the axial guide field is the dominant driver of the reconnection. The extent to which Hall currents can alleviate the buildup of back pressures in flux pile-up reconnection models is also examined. The analysis shows that, although enhancements of the merging rate can be expected under certain conditions, it is unlikely that Hall currents can completely undo the fundamental pressure limitations associated with flux pile-up reconnection. (C) 2005 American Institute of Physics. [DOI: 10.1063/1.1826094]
\end{abstract}

\section{INTRODUCTION}

Magnetic reconnection is a fundamental resistive process that accounts for topological change in magnetic plasmas. Since astrophysical and laboratory plasmas are highly conducting it is often difficult to construct reconnection mechanisms that are fast enough to explain observations of rapid magnetic collapse. This is a recurring problem in applying reconnection theory to real phenomena, for instance, the explosive energy release of solar flares.

In constructing reconnection models it has been customary to assume a resistive form of Ohm's law in which inertial effects and Hall currents are neglected. ${ }^{1}$ This long standing practice has been questioned in a recent series of analytic and computational studies. ${ }^{2-7}$ Hall currents, in particular, seem likely to have a strong influence on magnetic merging solutions.

The purpose of the present paper is to examine a family of exact analytic Hall reconnection models based on breaking the flow and field variables into background and disturbance components. These solutions extend earlier work, ${ }^{6,8}$ by including large scale background components in the perpendicular (axial) flow and magnetic fields. In fact we show that large scale axial guide components are naturally incorporated in a time-dependent MHD (magnetohydrodynamic) framework that includes Hall currents and inertial effects within a generalized Ohm's law for the plasma. Our present focus, however, is to examine how Hall currents affect reconnection rates and Ohmic dissipation rates, and for this purpose it is convenient to adopt a simplified analytic treatment in which time dependence, and viscous and inertial effects are neglected. We also address the issue of whether Hall currents

\footnotetext{
a)Electronic mail: math0097@waikato.ac.nz

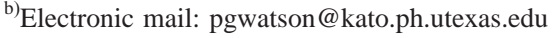

can alleviate the strong back pressures that limit the merging rates of flux pile-up reconnection solutions.

It is interesting to note that attempts have already been made to elude the pressure limitation of flux pile-up models. Jardine and Allen ${ }^{9}$ point out that the strength of the background velocity field can be adjusted to alleviate the strong build up of magnetic pressure in the current layer. Unfortunately, this remedy is compromised by the much stronger dynamic pressures required to sustain the merging. More recently, Dorelli ${ }^{10}$ has suggested that Hall currents, in combination with strong axial guide fields, can help ease the pressure problem. Dorelli's suggestion is consistent with numerical simulations, ${ }^{11,12}$ which imply that the form of the axial field (and specifically its gradient) is critical in determining the reconnection rate. Our results suggest that although Dorelli's prescription allows the pressure limitation on the reconnection rate to be eased somewhat, it is unlikely to be eliminated entirely unless a far more complicated background field structure is invoked.

The details of the general analytic description are given in Secs. II and III. In Sec. IV we demonstrate that Hall currents can both increase or decrease the reconnection rate by altering the thickness of the reconnecting current sheet. Speed up in the reconnection rate is achieved when the sheet thins, but this occurs at the expense of the Ohmic dissipation rate. In contrast, thicker sheets lead to slower reconnection rates, but higher Ohmic dissipation rates. Which type of solution results depends on the symmetries of the merging problem - in particular the sign of the axial guide field component. As regards the pressure problem, our analysis in Sec. $\mathrm{V}$ suggests that, for the simplified analytic models described by Dorelli ${ }^{10}$ and developed further here, Hall currents are limited in their ability to alleviate the pressure restrictions on flux pile up merging. It is possible, however, that the pressure problem could be eliminated in a more general 
approach-indeed numerical experiments ${ }^{11,12}$ suggest that the pileup can become independent of plasma resistivity without the amplitude of the axial field becoming too large. An alternative interpretation of the analytic results presented here, where it is the gradient of the axial field that scales strongly with $\eta$ rather than the amplitude, could lead to this type of behavior, but only at the expense of adding a second (undetermined) small length scale to the background field profiles of the model. This possibility is addressed in Secs. V and VI.

\section{MHD RECONNECTION EQUATIONS}

\section{A. The generalized Ohm's law}

We consider an incompressible magnetic plasma in which $\boldsymbol{\nabla} \cdot \mathbf{v}=\boldsymbol{\nabla} \cdot \mathbf{B}=0$. The governing equations are the dimensionless momentum and induction equations, in which magnetic fields, lengths, and particle densities are scaled according to typical reference values; velocities and time are measured in units of the Alfvén speed and the Alfvén time, respectively.

The dimensionless momentum equation takes the form

$$
\frac{\partial \mathbf{v}}{\partial t}+(\mathbf{v} \cdot \boldsymbol{\nabla}) \mathbf{v}=-\nabla p+\mathbf{J} \times \mathbf{B}+\nu \nabla^{2} \mathbf{v}
$$

where $\mathbf{J}=\boldsymbol{\nabla} \times \mathbf{B}$ is the current density, $\nu$ is the plasma viscosity, and $p$ is the plasma pressure. The induction equation is derived from the generalized Ohm's law

$$
\begin{aligned}
\mathbf{E}+\mathbf{v} \times \mathbf{B}= & \eta \mathbf{J}+d_{i}\left(\mathbf{J} \times \mathbf{B}-\boldsymbol{\nabla} p_{e}\right) \\
& +d_{e}^{2}\left(\frac{\partial \mathbf{J}}{\partial t}+\boldsymbol{\nabla} \cdot(\mathbf{v} \mathbf{J}+\mathbf{J v})\right),
\end{aligned}
$$

where we have replaced the tensor form for the electron pressure $\boldsymbol{\nabla} \cdot \mathbf{p}_{e}$ with the diagonal term $\boldsymbol{\nabla} p_{e}$, where $p_{e}$ is a scalar. Here

$$
\eta=\frac{c^{2}}{4 \pi v_{A} \ell \sigma}, \quad d_{i}=\frac{c}{\ell \omega_{p i}}, \quad d_{e}=\frac{c}{\ell \omega_{p e}},
$$

$\omega_{p e}$ and $\omega_{p i}$ are the electron and ion plasma frequencies $\omega_{p e}{ }^{2}=4 \pi n e^{2} / m_{e}=m_{p} \omega_{p i}{ }^{2} / m_{e}, c$ is the speed of light, $v_{A}$ is the Alfvén speed, $\sigma$ is the plasma conductivity, and $\ell$ is the typical plasma length scale. The dimensionless numbers $\eta$, $d_{i}$, and $d_{e}$ are, respectively, the inverse Lundquist number and the ion and electron skin depths, and indicate the relative magnitudes of the collisional, Hall, and inertial terms. The high conductivity of plasmas implies that the resistivity $\eta$ is very small: for example, typical values for a coronal plasma based on characteristic reference parameters are $\eta=10^{-14.5}$, $d_{i}=10^{-6.5}$, and $d_{e}=10^{-8}$.

\section{B. The planar reconnection equations}

In what follows solutions for the velocity and magnetic fields $\mathbf{v}$ and $\mathbf{B}$ are sought over the planar domain $-1 \leqslant x, y$ $\leqslant 1$, where $z$ is an ignorable coordinate. It is convenient to use stream and flux function representations for the $\mathbf{v}$ and $\mathbf{B}$ fields

$$
\mathbf{v}(x, y, t)=\boldsymbol{\nabla} \phi(x, y, t) \times \hat{\mathbf{z}}+\mathcal{W}(x, y, t) \hat{\mathbf{z}},
$$

$$
\mathbf{B}(x, y, t)=\boldsymbol{\nabla} \psi(x, y, t) \times \hat{\mathbf{z}}+\mathcal{Z}(x, y, t) \hat{\mathbf{z}} .
$$

In components we have that $\mathbf{v}=\left(\phi_{y},-\phi_{x}, \mathcal{W}\right)$ and $\mathbf{B}=\left(\psi_{y}\right.$, $\left.-\psi_{x}, \mathcal{Z}\right)$, where subscripted variables denote partial derivatives. Note that the current density

$$
\mathbf{J}=\left(\mathcal{Z}_{y},-\mathcal{Z}_{x},-\nabla^{2} \psi\right)
$$

comprises both axial and planar contributions.

In considering the advection of the magnetic field by the flow, it is useful to introduce the Poisson bracket notation typified by

$$
[\psi, \phi]=\psi_{x} \phi_{y}-\psi_{y} \phi_{x} .
$$

The curl of the momentum equation yields the planar components of the velocity field

$$
\nabla^{2} \phi_{t}+\left[\nabla^{2} \phi, \phi\right]=\left[\nabla^{2} \psi, \psi\right]+\nu \nabla^{4} \phi,
$$

while

$$
\mathcal{W}_{t}+[\mathcal{W}, \phi]=[\mathcal{Z}, \psi]+\nu \nabla^{2} \mathcal{W}
$$

gives the axial component.

The curl of Ohm's law yields the magnetic induction equation. The third component reduces to

$$
\begin{aligned}
\psi_{t}+[\psi, \phi]= & \eta \nabla^{2} \psi+d_{i}[\psi, \mathcal{Z}] \\
& +d_{e}^{2}\left(\nabla^{2} \psi_{t}+\left[\nabla^{2} \psi, \phi\right]+[\mathcal{Z}, \mathcal{W}]\right)
\end{aligned}
$$

while the first and second components determine the axial field

$$
\begin{aligned}
\mathcal{Z}_{t}+[\mathcal{Z}, \phi]= & \eta \nabla^{2} \mathcal{Z}+[\mathcal{W}, \psi]+d_{i}\left[\nabla^{2} \psi, \psi\right] \\
& +d_{e}^{2}\left(\nabla^{2} \mathcal{Z}_{t}+\left[\nabla^{2} \mathcal{Z}, \phi\right]+\left[\nabla^{2} \phi, \mathcal{Z}\right]\right)
\end{aligned}
$$

This system completely determines the planar reconnection problem.

\section{Hall current contributions}

Of central interest is whether Hall currents can lead to enhanced magnetic merging rates. Before specializing to particular reconnection solutions, it is instructive to make some preliminary observations based on the general system of planar reconnection equations introduced above.

First note that the evolution equation for $\psi$ can be written in a form that manifests the modified advection provided by the Hall term

$$
\psi_{t}+\left[\psi, \phi-d_{i} \mathcal{Z}\right]=\eta \nabla^{2} \psi+d_{e}^{2}\left(\nabla^{2} \psi_{t}+\left[\nabla^{2} \psi, \phi\right]+[\mathcal{Z}, \mathcal{W}]\right)
$$

The change in the advection bracket effected by the axial guide field, $[\psi, \phi] \rightarrow\left[\psi, \phi-d_{i} \mathcal{Z}\right]$, reflects the fact that the $\mathbf{B}$ field is advected by the electron fluid, rather than the massaveraged velocity of the electron-proton gas. Whether the Hall term speeds up or slows down the convective transport clearly depends on the properties of the axial field $\mathcal{Z}$.

In fact, as detailed in the Appendix, there are two distinct components to the axial field. One component, the large scale "guide" component, is independent of the reconnection dynamics, while the other component is induced by the reconnection of the planar field. The induced field follows 
from Eq. (9), where it is clear that even if $\mathcal{Z}=0$ initially, the presence of the term $d_{i}\left[\nabla^{2} \psi, \psi\right]$ will drive a growth in $\mathcal{Z}$. This field may, in turn, feed back into the advection of the planar reconnecting field altering the reconnection rate. The induced axial field also adds planar components to the current density $\mathbf{J}$, which can lead to an increase in the Ohmic dissipation rate

$$
W_{\eta}=\eta \int J^{2} d V=\eta \int\left[\left(\nabla^{2} \psi\right)^{2}+\mathcal{Z}_{x}^{2}+\mathcal{Z}_{y}^{2}\right] d V .
$$

Although the presence of an axial guide component depends only on the initial conditions of the problem, it can, depending on its magnitude and orientation, lead to significant changes in the reconnection rate. These possibilities are discussed in detail below.

\section{INFLUENCE OF THE HALL CURRENT}

\section{A. Introduction}

We now turn to a class of reconnection solutions that makes explicit the division between guide fields and reconnecting field components. In the Appendix it is shown that time-dependent reconnection solutions can be developed from the planar MHD equations using a moment expansion technique. The essential point is that the resulting system can be interpreted in terms of localized reconnection fields that are superposed on large scale background guide fields. In particular, the stagnation flow potential $\phi=-\alpha x y$ can be used to provide a prototype guide field.

The simplest exact solution, of sufficient generality for our purposes, is obtained by taking $\partial_{t}=\nu=d_{e}=0$. The potentials are given by

$$
\begin{aligned}
& \phi(x, y)=-\alpha x y+f(x), \\
& \psi(x, y)=\beta x y+g(x),
\end{aligned}
$$

together with the axial fields

$$
\begin{aligned}
& \mathcal{W}(x, y)=\gamma x y+W(x), \\
& \mathcal{Z}(x, y)=\delta x y+Z(x) .
\end{aligned}
$$

Provided that we take $\alpha>0$ to maintain a global inflow, these forms describe steady-state reconnection maintained by a one-dimensional (1D) current sheet aligned to the $y$ axis.

There is, however, an important constraint on the solution that derives from the condition that the plasma pressure must satisfy $p(x, y)>0$. The pressure is determined by integrating the separate components of the inviscid momentum Eq. (1). The exact expression, valid throughout the solution domain $-1<x, y<1$, can be written as

$$
\begin{aligned}
p(x, y)= & p_{0}-\frac{1}{2}\left[\alpha^{2}\left(x^{2}+y^{2}\right)+g^{\prime}(x)^{2}+\mathcal{Z}(x, y)^{2}\right] \\
& -\beta y g^{\prime}(x) .
\end{aligned}
$$

Clearly, since the background pressure amplitude $p_{0}$ is bounded, the flow and field magnitudes $\alpha,\left|g^{\prime}(x)\right|$, and $|\mathcal{Z}(x, y)|$ cannot be too large if the pressure is to remain positive. It is this constraint that eventually limits the merging rate of flux pile-up reconnection models. ${ }^{13-15}$

\section{B. The disturbance fields}

Solutions for the disturbance fields are derived by substituting forms (12)-(15) into the planar reconnection equations. To within a nonessential quadratic function ${ }^{8}$ we find from (6) that

$$
f=-\frac{\beta}{\alpha} g,
$$

while (7) yields

$$
W=-\frac{\beta}{\alpha} Z+\frac{1}{\alpha}\left(\delta+\frac{\beta \gamma}{\alpha}\right) g .
$$

The planar components of the induction equation now reduce to

$$
\mathcal{E}-\left(\alpha^{*}+d_{i} \delta\right) x g^{\prime}=\eta g^{\prime \prime}-\beta d_{i} x Z^{\prime},
$$

where $\alpha^{*}=\left(\alpha^{2}-\beta^{2}\right) / \alpha$ and $\mathcal{E}$ is the steady state flux transfer rate $\psi_{t}$. The axial component is given by

$$
-\alpha^{*} x Z^{\prime}=\eta Z^{\prime \prime}-\frac{\gamma}{\alpha} \alpha^{*} x g^{\prime}+\beta d_{i} x g^{\prime \prime \prime} .
$$

These equations must be simultaneously resolved for $g$ and $Z$ to obtain reconnection solutions. Craig and Watson ${ }^{6}$ have already considered the case in which the axial guide fields vanish $(\gamma=\delta=0)$, while Dorelli ${ }^{10}$ considers head-on merging $(\beta=0)$ in the presence of finite axial fields. Below we examine the more general case of steady-state sheared reconnection in which all guide field components are present.

\section{Equation for the planar disturbance field}

By eliminating the axial field $Z(x)$ it is possible to obtain a single differential equation for the disturbance potential $g(x)$. On introducing the variables

$$
s=\left(\frac{\alpha^{+}}{\eta}\right)^{1 / 2} x, \quad \kappa=\frac{\beta^{2} d_{i}^{2}}{\alpha^{+} \eta}, \quad \alpha^{+}=\alpha^{*}+\delta d_{i},
$$

we obtain

$$
\left(s+\kappa s^{3}\right) G^{\prime \prime}+\left[(1+A) s^{2}-1\right] G^{\prime}+B s^{3} G=A s^{2}-1,
$$

where

$$
A=\frac{\alpha^{*}}{\alpha^{+}}, \quad B=\frac{\alpha^{*}}{\alpha^{+}}\left(1-\frac{\beta \gamma d_{i}}{\alpha \alpha^{+}}\right), \quad G(s)=\frac{\alpha^{+}}{\mathcal{E}} g^{\prime}(s) .
$$

Several key points emerge from Eq. (22). First note that the equation is written in a form that isolates the axial guide field amplitudes $\gamma$ and $\delta$ in the coefficients $A$ and $B$. If the axial guide fields vanish then $A=B=1$ and the solution contains a single parameter $\kappa$ that measures the competing effects of the Hall current and the resistivity. ${ }^{6}$ If we also let $\kappa \rightarrow 0$, then our rescaling filters out all the parameters from the equation, and it is clear that the resistively dominated solution must possess a single small length scale $s=O(1)$, or in terms of our original variables $x \simeq \ell=\left(\eta / \alpha^{+}\right)^{1 / 2}$. This scale, in separating the outer advection region from the current sheet that sustains the merging, defines both the sheet thickness and the reconnection speed $\eta / \ell \simeq\left(\eta \alpha^{+}\right)^{1 / 2}$. 
The equation is more complicated when we have axial guide fields, but we still expect the coefficient of the highest derivative to have a major impact on the solution. This suggests qualitative changes should be apparent when

$$
\kappa=\frac{\beta^{2} d_{i}^{2}}{\left(\alpha^{*}+\delta d_{i}\right) \eta}>1 .
$$

In addition, there are various conditions on $A$ and $B$ that must be satisfied if we wish to obtain physically relevant solutions. For example if $\kappa>0$ and $A>0$ we require $B>0$ to get localized solutions.

\section{ANALYTIC HALL CURRENT SOLUTIONS}

Although it is not possible to obtain a general analytic solution of (22), many of the essential features can be brought out by examining special cases. These examples clarify much of reconnection physics, as well as highlighting some inherent difficulties. We begin by summarizing the case in which the axial guide fields are turned off.

\section{A. Hall MHD with no axial guide fields: $\gamma=\delta=0$}

In the case $\gamma=\delta=0 \mathrm{Eq}$. (22) reduces to

$$
\left(s+\kappa s^{3}\right) G^{\prime \prime}+\left(2 s^{2}-1\right) G^{\prime}+s^{3} G=s^{2}-1,
$$

and we must take $\alpha^{+}=\alpha^{*}$ in (23). Solutions to this problem are developed in the work Craig and Watson, ${ }^{6}$ and we present only a summarized discussion here.

First note that competing effects of the Hall current and resistivity are combined in the single parameter $\kappa \sim d_{i}^{2} / \eta$. When this parameter is small the problem is resistively dominated and for $\kappa<1$ the solutions are found to display only very modest departures from the purely resistive $(\kappa$ $=0)$ solution. Hall effects begin to dominate once $\kappa>1$, and oscillatory behavior develops outside the primary reconnection layer. Once $\kappa$ becomes much larger than unity the solution reverts to a simple standing whistler wave, with a wavelength proportional to $d_{i}$.

The key results in this regime are that the Hall effect thickens the sheet and increases the peak magnetic field. These effects are an artifact of the Hall coupling between the axial disturbance field $Z$ and the planar reconnecting field $g$. In the context of saturated solutions, ${ }^{14,15}$ where the peak field is limited to a maximum value dictated by the driving hydromagnetic pressures exerted at the boundaries, these results imply a slowing of the reconnection rate. Although the reconnection rate slows for large values of the Hall parameter, the appearance of oscillations leads to marked increases in the Ohmic dissipation rate due to the presence of multiple current sheets and the increased contributions of the growing axial field to the Ohmic dissipation rate, see (11).

These results are at odds with the findings of many numerical simulations of Hall MHD that observe marked increases in the reconnection rate as the Hall effect begins to dominate. ${ }^{3,5,16,17}$ Although the significantly enhanced reconnection rates observed in these experiments could be an artifact of transient effects, we believe that a more likely explanation lies in the differing symmetries assumed in the analytic and numerical merging solutions. As shown below, the inclusion of a suitably oriented axial guide field can lead to solutions in which the current sheet is thinned and the reconnection rate is appreciably enhanced.

\section{B. Hall MHD annihilation with axial guide field: $\beta=0$}

We now consider a simple example where we retain the axial guide fields $(\gamma, \delta \neq 0)$, but let $\beta \rightarrow 0$. This type of solution corresponds to the "head-on" annihilation of straight field lines in the reconnection plane and is the case considered by Dorelli. ${ }^{10}$ In this limit $\kappa=0, \alpha^{*}=\alpha, \alpha^{+}=\alpha+\delta d_{i}$ and Eq. (22) reduces to

$$
s G^{\prime \prime}+\left[\left(1+\frac{\alpha}{\alpha^{+}}\right) s^{2}-1\right] G^{\prime}+\frac{\alpha}{\alpha^{+}} s^{3} G=\frac{\alpha}{\alpha^{+}} s^{2}-1 .
$$

This equation has the solution

$$
G(s)=\sqrt{2} \operatorname{daw}\left(\frac{s}{\sqrt{2}}\right),
$$

where we have retained only the odd (reconnecting) part of the general solution and $\operatorname{daw}(z)$ is the Dawson function,

$$
\operatorname{daw}(z)=e^{-z^{2}} \int_{0}^{z} e^{t^{2}} d t
$$

In terms of the original variables we have

$$
g^{\prime}(x)=\frac{E}{\eta \mu} \operatorname{daw}(\mu x), \quad \mu^{2}=\frac{\alpha+\delta d_{i}}{2 \eta}
$$

[in fact it is simpler to derive this solution directly from (19) with $\beta=0$ ]. Although we may solve (20) for $Z(x)$, we see that it does not feed back on the planar field in this case. This component cannot affect the reconnection rate but it will modify the Ohmic dissipation rate, as discussed in Sec. II C.

As emphasized by Dorelli, ${ }^{10}$ we note that solution (28) identifies

$$
\ell \simeq\left(\frac{\eta}{\alpha+\delta d_{i}}\right)^{1 / 2}
$$

as the thickness of the current sheet centered on $x=0$. The influence of the Hall term in this example is to enhance the electron inflow speed (provided $\delta>0$ ) leading to a thinner sheet and an enhanced reconnection rate. However, we could equally well choose $\delta<0$ to effect a broadening of the sheet and a reduction in the merging rate. This demonstrates the point made in Sec. IV A above, namely, the sensitivity of the solution to the symmetry properties of the axial guide field in the Hall MHD limit.

Irrespective of whether we thin or broaden the sheet, a significant modification of the solution due to the Hall mechanism requires $\delta d_{i} \gtrsim \alpha$. Since $\alpha$ defines the strength of the driving flow it is expected to be of order unity in a fast reconnection mechanism where Alfveníc exhausts are anticipated, and dynamic and magnetic pressures are expected to be comparable. Remembering that $d_{i} \ll 1$ for a typical plasma we see that $\delta \gg 1$ is required to achieve an appreciable effect. The implication-unless the veracity of the alternative interpretation of Sec. V can be demonstrated-is that a large axial guide field and consequently high magnetic pressures are 
required. We conclude that changes in the reconnection rate are likely to be minor unless the flow magnitude is itself very weak, that is, $\alpha \ll 1$.

\section{Axial field driven reconnection: $\alpha^{\star} / \alpha^{+} \rightarrow 0$}

Another case where we can make some analytic progress is the limit $\alpha^{*} / \alpha^{+} \rightarrow 0$ (either by assuming $\alpha^{*}=0$ or $\alpha^{*}$ $\left.\ll \alpha^{+}\right)$. Now we arrive at a regime where the reconnection is driven purely by the contribution of the axial guide field to the electron velocity $\alpha^{+} \simeq \delta d_{i}$ (note we must now fix $\delta>0$ to ensure inflow of the field carrying electrons). In this case (22) reduces to

$$
\left(s+\kappa s^{3}\right) G^{\prime \prime}+\left(s^{2}-1\right) G^{\prime}=-1,
$$

and the solution can be expressed as a quadrature of the form

$$
G(s)=\int_{0}^{s}\left(1+\kappa u^{2}\right)^{-(1+\kappa) / 2 \kappa}{ }_{2} F_{1}\left(-\frac{1}{2}, \frac{\kappa-1}{2 \kappa} ; \frac{1}{2} ;-\kappa u^{2}\right) d u .
$$

When $(\kappa-1) /(2 \kappa)$ is a negative integer, the hypergeometric function can be expressed as a terminating series, making closed form solutions possible. The first few values of $\kappa$ are

$$
\begin{aligned}
& \kappa=1: \quad G=\tan ^{-1}(s), \quad Z=\frac{\mathcal{E}}{\beta d_{i}}\left[s \tan ^{-1} s-\ln \left(s^{2}+1\right)\right]+Z_{0} ; \\
& \kappa=\frac{1}{3}: \quad G=\frac{3 s}{s^{2}+3}, \quad Z=\frac{\mathcal{E}}{\beta d_{i}}\left[-\frac{s^{2}}{s^{2}+3}+\ln \left(\frac{s^{2}+3}{3}\right)\right]+Z_{0} ; \\
& \kappa=\frac{1}{5}: \quad G=\frac{5 s\left(s^{2}+15\right)}{3\left(s^{2}+5\right)^{2}}, \\
& Z=\frac{\mathcal{E}}{\beta d_{i}}\left[\frac{s^{2}\left(s^{2}-5\right)}{3\left(s^{2}+5\right)^{2}}+\frac{1}{3} \ln \left(\frac{s^{2}+5}{5}\right)\right]+Z_{0} ; \\
& \kappa=\frac{1}{7}: \quad G=\frac{7 s\left(3 s^{4}+70 s^{2}+735\right)}{15\left(s^{2}+7\right)^{3}}, \\
& Z=\frac{\mathcal{E}}{\beta d_{i}}\left[\frac{s^{2}\left(5 s^{4}+70 s^{2}-147\right)}{15\left(s^{2}+7\right)^{3}}+\frac{1}{5} \ln \left(\frac{s^{2}+7}{7}\right)\right]+Z_{0} ; \\
& \vdots \\
& \kappa=0: \quad G=\sqrt{2} \mathrm{daw}\left(\frac{s}{\sqrt{2}}\right), \quad Z=Z_{0} ;
\end{aligned}
$$

where the solutions for $Z$ are obtained from (20) by setting $\alpha^{*}=0$ and remembering $\kappa=\beta^{2} d_{i} /(\delta \eta)$.

Figures 1(a) and 1(b) show plots of the fields $G(s)$ and $Z(s)$ for these solutions. There are several features to note. First, the change in the qualitative structure of the planar field $G(s)$ is relatively modest as we vary $\kappa$ from $\kappa=0$ (the resistively dominated limit) to $\kappa=1 / 3$. However, once $\kappa$ approaches unity the solution undergoes a fundamental change and the localized nature of the solution breaks down entirely. Also, the axial disturbance field $Z$ has a weak logarithmic (a)

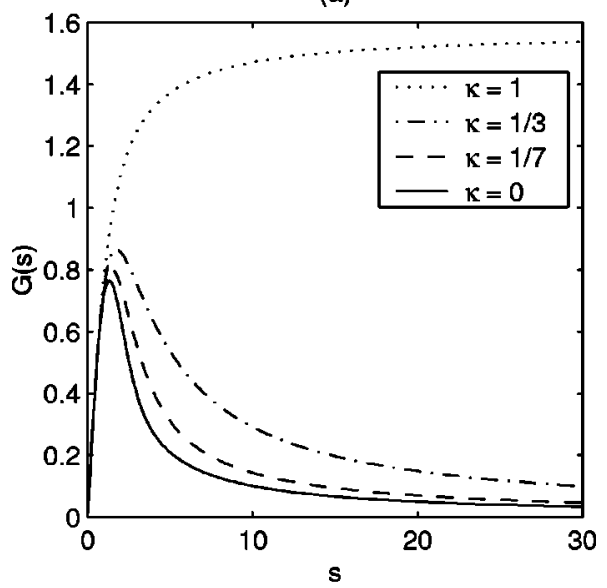

(b)

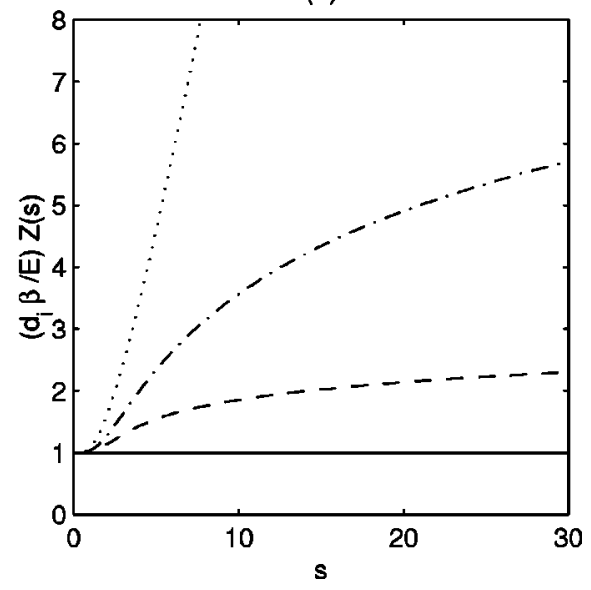

FIG. 1. Magnetic disturbance fields for axial field driven reconnection with $\alpha^{*} / \alpha^{+} \rightarrow 0$. (a) The planar disturbance field $G(s)$ vs $s$ for various values of $\kappa$. There is little qualitative change in the structure of the solution in going from $\kappa=0$ to $\kappa=1 / 3$, however, as $\kappa$ approaches unity the field peak moves out to infinity and $G$ is no longer localized. Note, the apparent thickening of the sheet as $\kappa$ is increased is a somewhat misleading artifact of our rescaling. The true behavior of the width of the sheet in terms of the physical variables is more complicated. (b) The corresponding normalized axial disturbance fields $\left(\beta d_{i} / \mathcal{E}\right) Z(s)$ vs $s$. These solutions for $Z$ have a weak logarithmic growth away from the sheet for $\kappa<1$, but once $\kappa \geqslant 1$ they begin to grow linearly or faster.

growth away from the reconnecting current layer. This implies that for this type of steady solution, the largest contribution to the magnetic pressure from the axial field occurs well away from the current sheet.

Looking at Fig. 1(a) we might assume that the sheet thickness increases monotonically as $\kappa$ is increased-once again implying a decreasing reconnection rate as the effect of the Hall current is increased. This is not actually the case, as our rescaling of the problem is obscuring the true behavior of the sheet width with varying $\kappa$.

The true situation is somewhat more complicated since it depends on competing Hall effects represented by the induced and guide components of the axial field. To see this it is useful to define the sheet width as the location of the maximum of $G(s)$ : we have that 


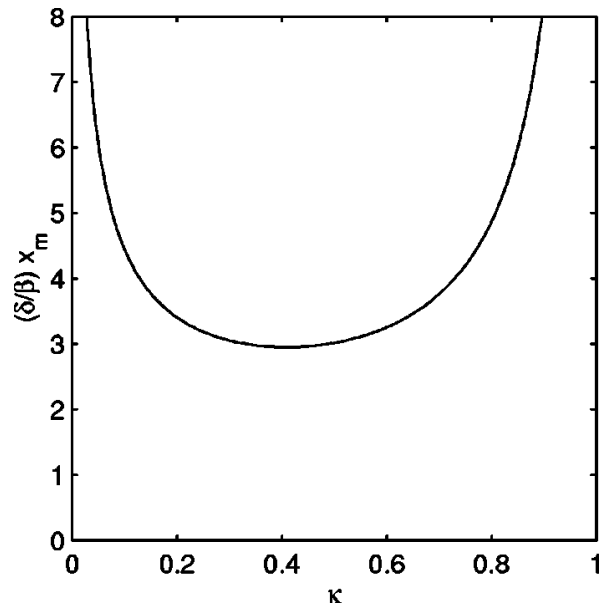

FIG. 2. A plot of the normalized sheet width vs $\kappa$ for axial guide field driven reconnection. If the parameters $\alpha, \beta, \gamma, \delta$, and $\eta$ are fixed then increasing $\kappa$ corresponds to increasing $d_{i}$. If $\kappa$ and $d_{i}$ are small then the effective inflow $\alpha^{+}=\delta d_{i}$ is weak and the sheet is broad. Increasing $\kappa$ strengthens the inflow and this initially leads to thinner sheets. However, a point is reached near $\kappa=0.4$, where the competing effect of the coupling of the induced axial disturbance field takes over and it begins to thicken the sheet.

$$
\ell=x_{m}=\left(\frac{\eta}{\alpha^{+}}\right)^{1 / 2} s_{m}=\left(\frac{\eta}{\delta d_{i}}\right)^{1 / 2} s_{m}=\left(\frac{\beta^{2}}{\kappa \delta^{2}}\right)^{1 / 2} s_{m}
$$

where $x_{m}$ and $s_{m}$ are the positions of the maximum of $G$ in terms of the $x$ and $s$ variables, respectively. However, $s_{m}$ is itself a function of $\kappa$ [see Fig. 1(a)] that we can obtain by solving

$$
{ }_{2} F_{1}\left(-\frac{1}{2}, \frac{\kappa-1}{2 \kappa} ; \frac{1}{2} ;-\kappa s_{m}^{2}\right)=0
$$

A plot of (normalized) $x_{m}$ versus $\kappa$ is shown in Fig. 2. We now see that the sheet width initially decreases (and the reconnection rate increases) as $\kappa$ is increased from zero, until $x_{m}$ reaches a minimum around $\kappa=0.4$. After that $x_{m}$ begins to increase again (accompanied by a decrease in the reconnection rate), finally tending to infinity as $\kappa \rightarrow 1$.

Recall that the Hall term makes its presence felt through the axial magnetic field $\mathcal{Z}(x, y)=\delta x y+Z(x)$ in two distinct ways. First, the guide field component $\delta x y$ acts to increase the effective electron inflow velocity (assuming $\delta>0$ ), while at the same time the disturbance component $Z(x)$ couples to the reconnecting planar field $g$. These effects were isolated in the discussions of Secs. IV A and IV B, but now compete for dominance. If we fix $\alpha, \beta, \gamma, \delta$, and $\eta$, then increasing $\kappa$ is equivalent to increasing $d_{i}$. Now it is clear that increasing $\kappa$ corresponds to increasing the effective electron inflow velocity $\alpha^{+}=\delta d_{i}$, which leads to thinner sheets and faster reconnection. The direct coupling between $Z$ and $g$ tends to have the opposite effect, leading to broadened profiles for the planar field $G$. Therefore the solutions in this case show a mixture of the different behaviors of the solutions outlined in Secs. IV A and IV B above.

\section{PRESSURE LIMITATIONS IN FLUX PILE-UP MERGING}

The chief problem with the reconnection solutions of the preceding section is the massive field intensities at the current sheet predicted by the various models. The magnitude of the planar field at the onset of the current sheet for the models outlined in Secs. IV B and IV $\mathrm{C}$ above is given by

$$
B_{s} \equiv\left|g^{\prime}(x=\ell)\right| \sim \frac{\mathcal{E}}{\left(\eta \alpha^{+}\right)^{1 / 2}}, \quad \alpha^{+}=\frac{\alpha^{2}-\beta^{2}}{\alpha}+\delta d_{i},
$$

remembering that

$$
g^{\prime}(x)=\frac{d s}{d x} g^{\prime}(s)=\left(\frac{\alpha^{+}}{\eta}\right)^{1 / 2} \frac{\mathcal{E}}{\alpha^{+}} G(s)=\frac{\mathcal{E}}{\left(\eta \alpha^{+}\right)^{1 / 2}} G(s)
$$

and noting that the peak value of $G(s)$ is $O(1)$.

Suppose, for example, that the reconnection rate $\mathcal{E}$ is held fixed as $\eta$ is systematically reduced. Then to maintain the reconnection rate-assuming all other parameters are also held constant-requires a huge flux pile-up field $B_{s}$ $\simeq \eta^{-1 / 2}$. The build up in pressure is even more dramatic-as we require the background pressure $p_{0}$ to satisfy $p_{0}>\frac{1}{2} B_{s}^{2}$ $\sim \eta^{-1}$ according to (16).

One possibility to keep the pressure at physically realistic levels is to limit the size of magnetic disturbances washed into the reconnection region. Basically, the disturbance field on the outer boundary of the advection region $x \simeq 1$ must always be small enough to maintain plausible values for $B_{s}$. It is this constraint on the disturbance field amplitude that limits the merging rate of flux pile-up solutions to physically plausible levels. Above this level, the current sheet pressure is sufficient to stall the inflow, saturating the reconnection rate.

In view of the historical importance attached to fast reconnection solutions in the formal limit $\eta \rightarrow 0$, it is not surprising that attempts have been made to overcome the pressure restriction without saturating the reconnection rate. Two papers in particular, by Jardine and Allen ${ }^{9}$ and by Dorelli, ${ }^{10}$ deserve special mention. Although these two papers deal with very different problems and are motivated by different considerations, they do share a common theme in that the flux pileup observed in the planar field near the current sheet can be eased by a suitable modification of the merging mechanism. Jardine and Allen ${ }^{9}$ deal with a purely resistive MHD merging problem. Their main concern is to develop nonzero vorticity solutions, where the global length scale of the background flow can be self-consistently determined. However, their work implies that the flow amplitude can be scaled (by adjusting the vorticity at the inflow boundaries) so that $\alpha=\alpha_{0} \eta^{-1}$ in order to ease the planar magnetic pressure buildup. As (35) with $\beta=\delta=0$ confirms, this does indeed eliminate the build up of $B_{s}$, but only at the expense of massive dynamic pressures $\rho v^{2} \sim \eta^{-2}$ in the background flow. ${ }^{18}$

More recently, Dorelli ${ }^{10}$ has investigated magnetic merging in the context of Hall MHD. His solutions (and those presented here) differ significantly from Jardine and Allen's in that the background flow is vorticity-free and hence the global scale of the background flow and field is not uniquely determined. In common with Jardine and Allen, Dorelli con- 
cludes that it is possible to mitigate the pressure constraint imposed on the planar field - in his case by using the axial guide field to thin down the current sheet width. However, as can be seen from (35), the axial guide field must have the scaling $\delta=\delta_{0} \eta^{-1}$ if flux pileup is to be completely eliminated. This huge guide component $\mathcal{Z}(x, y)=\delta x y$ does not appear in Dorelli's expression for the pressure, which is restricted solely to the inflow axis $y=0$. But substituting for $\mathcal{Z}=\delta x y$ in (16), confirms that a severe constraint on the pressure still persists away from the coordinate lines $x=y=0$.

It seems clear, therefore, that Dorelli's suggestion, along with Jardine and Allen's, simply transfers the pressure associated with the reconnecting field component into the flow and axial guide fields. In practice, it appears that the best strategy is to weight uniformly the individual contributions to the static and dynamic pressures in Eq. (16) - in other words scale the background flow and axial field field strengths so that they mimic the build-up of the reconnecting field $B_{s}$. This requires that both $\alpha$ and $\delta$ scale as $B_{s}$, which from (35) with $\mathcal{E}$ fixed, leads to the build-up $B_{s} \simeq \mathcal{E} \eta^{-1 / 3}$. Note that in the case of negligible guide field the prescription $\alpha \simeq B_{s}$ accords with the classical Sweet-Parker model-that is, the exhaust speed of material ejected from the sheet is determined by the Alfvén speed associated with the magnetic field $B_{s}$ at the onset of the current layer. ${ }^{14,15,18}$ This does not eliminate the pressure problem altogether, but according to (35) it moderates the build up of the sheet field $B_{s}$ leading to thinner sheets, $\ell \sim \eta^{2 / 3}$, and faster inflows. Such scalings have been repeatedly observed in the presaturation regimes of resistive MHD magnetic reconnection simulations. ${ }^{19,20}$

As a final note we point out that associating $\delta$ with the magnitude of the axial field depends on the fact that we have normalized the lengths in our model such that the background field and flow are based on a global length scale. However, as $\delta$ multiplies the hyperbolic $x y$ term it really represents the gradient of the axial field. If the length scale of the domain is considered to be microscopic rather than macroscopic, then clearly the $\delta x y$ contribution to the pressure near the off-axis corners of the domain will be much smaller and will therefore lead to much less severe pressure constraints. The drawback with this interpretation is that there is no way to determine this second microscopic scale of the solution within the context of the present model. Even so, this modification could provide a link between the formally exact solutions presented here, and in Dorelli, and numerical simulations that suggest resistivity independent reconnection rates in the Hall dominated regime. ${ }^{3,5,11,12}$

\section{MORE GENERAL SOLUTIONS AND THE BREAKDOWN OF THE ANALYTIC MODEL}

In this paper we have only examined a subset of the possible solutions of the full governing Eq. (22). These solutions show, however, that the addition of axial guide components to the magnetic and velocity fields introduces a different level of complexity to the Hall reconnection solutions first presented in Craig and Watson. ${ }^{6}$ Although it is not possible to write down a closed form analytic general solution to (22), it is a simple matter to integrate the equation numeri- cally. However, it seems likely that the special cases considered above contain most of the basic features: in particular, they pinpoint the competing effects of the coupling of the guide and disturbance components of the axial magnetic field to the planar field.

An important question concerns the relevance of the analytic models to reconnection in physically realizable configurations. Note that the clear separation of the magnetic and velocity fields into disturbance and guide components is an analytic artifact that is seldom achievable in magnetic reconnection simulations. Specifically, since the nonlinear feedback of the reconnecting magnetic field on the velocity inflow is not represented in the analytic solution, it is impossible to capture the complicated transient behavior associated with the nonlinear saturation of the current sheet. ${ }^{13}$ Although saturation effects could, in principle, be modeled by retaining higher moments in the expansion of the solution, such an approach seems unprofitable given the complexities of the moment system.

To our knowledge detailed studies of the saturated current layer have been performed only for magnetic merging in the classical, resistive MHD approximation. In such cases the key assumption of a quasi-one-dimensional current layer still holds good, and resistive scaling laws based on the analytic model-assuming a saturated level for the magnetic field in the sheet—can still be applied for quantifying the bulk energetics. ${ }^{6,13}$ To what extent the inclusion of a generalized Ohm's law, or a truly collisionless description of the reconnecting current layer, modifies these conclusions is largely unknown at present. Related work on reconnection in weakly collisional plasmas with strong guide fields ${ }^{21}$ suggests that in some regimes the large scale quasi-one-dimensional sheets can become unstable. This transition may signal the switch over from one-dimensional current sheets to the cross shaped current structures observed in Hall MHD reconnection. 5,16,22 Watson and Porcelli ${ }^{21}$ also find that the effects of electron inertia can postpone, or even eliminate, saturation. These findings suggest that there remains considerable scope for investigating, analytically and numerically, the interplay between weakly collisional current sheets and the large scale advection region that supports the merging.

\section{CONCLUSIONS}

We have considered the impact of Hall currents on planar models of current sheet reconnection. We began by noting that current sheet models based on a generalized Ohm's law can be interpreted as a superposition of transient reconnecting components onto large scale background guide fields (see the Appendix). A simplified analytic description confirms that the influence of the Hall term is intrinsically linked to the symmetries of the reconnection geometry and to the orientation of axial guide fields. As a consequence, Hall current reconnection allows a far richer spectrum of magnetic merging solutions than classical resistive MHD.

Suppose, for instance, that the axial guide components are turned off. Then the reconnection of the planar field induces axial disturbance field components, which, in turn, feed back on the planar solution and thicken up the current 
sheet. This leads to enhanced Ohmic dissipation but only at the cost of diminishing the reconnection rate (see Sec. IV A).

When axial guide fields are present the picture is more complicated. The analysis of Secs. IV B and IV C shows that the guide field can, depending on its orientation, act to increase or decrease the effective advection of the planar field. If the advection is enhanced then the influence of the guide field will tend to increase the reconnection rate. However, the direct coupling of the axial disturbance field $Z$ to the planar field $g$ is still present and this tends to inhibit the merging. In general these two effects compete-and which one ultimately wins depends critically on the parameters of the merging problem at hand.

Given that the strength of the guide component is probably limited by pressure constraints, the guide field enhancement of the inflow is likely to be important only if the advection of the planar field by the global velocity field is weak. This might occur during the early phases of a resistive instability - in the tearing mode, say, prior to the build up of strong fluid velocities - or perhaps in strongly anisotropic configurations, such as the reconnection of weak poloidal field in a toroidally dominated system.

To date the majority of numerical simulations of Hall MHD have indicated that Hall effects speed up reconnection. In this paper we have shown that the real picture may be far more complicated. More generally, for instance in 2D timedependent simulations, the distinction between guide field and disturbance components cannot be sensibly maintained: the reconnecting fields eventually build up sufficiently to modify the background flow. However, the effect of different symmetries on the merging rate has been confirmed in a series of numerical experiments. ${ }^{7}$ It was observed that strong shear flows across the reconnection layer induced large axial fields that thicken the current sheet and slow the reconnection rate. In contrast, in head-on reconnection the current sheet became thinner and the merging rate was enhanced (at the expense of the Ohmic dissipation).

It is also possible to add the effects of electron inertia and time dependence into the model. These effects have already been examined in the related problem of weakly collisional reconnection in the limit of a strong guide field. ${ }^{21}$ In this problem there are two main regimes: a regime where finite Larmor radius effects dominate, and growing oscillatory solutions, much like those found in Craig and Watson, ${ }^{6}$ develop; and an electron inertia dominated regime, where a single peak localized solution naturally saturates. Watson and Porcelli $^{21}$ found that the oscillatory solutions become unstable, and the same is probably true of the oscillatory solutions found in the work by Craig and Watson ${ }^{6}$ and discussed here in Sec. IV A. However, the electron inertia dominated solutions do remain stable. The implication is that quasi-onedimensional reconnection-the classical current sheet model-may break down in certain collisionless limits.

Finally, we addressed the question of whether Hall currents can alleviate the buildup of strong hydromagnetic pressures in flux pile-up reconnection. In agreement with Dorelli, we find that flux pileup in the planar field can be be eliminated by invoking a sufficiently strong axial guide field. However, this solution is compromised by the strong pres- sure of the guide field away from the coordinate axes. In the model presented here the best that can be achieved is to balance the advection of the planar field, governed by the electron fluid, to the build up in magnetic pressure in the sheet. As discussed in Sec. V, this prescription moderates the pileup, $B_{s} \sim \eta^{-1 / 3}$, and leads to faster inflows and considerably thinner current sheets, $\ell \sim \eta^{2 / 3}$, in the presaturation phase of the merging. Alternatively we might interpret $\delta$ as the gradient of the axial field and consider that our solution is only valid on a microscopic region that straddles the reconnection region. This can dramatically ease the pressure constraint if the region size is small enough, but since we have no way of determining this microscopic scale from the analytic solution itself, the scale must be set a priori from external considerations. Under this interpretation we are left with the problem of welding our analytic "inner" Hall current solution onto a large scale outer solution, which does not significantly affect the merging dynamics. Although this might be a formidable task, it could provide a useful link between analytic Hall current models, such as that presented here, and the numerical simulations of Hall MHD reconnection.

\section{ACKNOWLEDGMENT}

One of the authors (I.J.D.C.) was partially supported by the Marsden Fund (Grant No. UOW201).

\section{APPENDIX A: MOMENT EXPANSION SOLUTIONS}

Consider the planar system of Sec. II B. If reconnection is to be effective then, in view of the smallness of the resistivity, current sheets involving small length scales must develop. If the current layer is such that $\partial_{x} \gg \partial_{y}$ then it is natural to consider an expansion of the form

$$
\left(\begin{array}{c}
\phi \\
W \\
\psi \\
Z
\end{array}\right)=\sum_{n=0}^{\infty} \frac{y^{n}}{n !}\left(\begin{array}{c}
\phi^{n}(x, t) \\
W^{n}(x, t) \\
\psi^{n}(x, t) \\
Z^{n}(x, t)
\end{array}\right)
$$

in which low order components provide the dominant contributions.

Two key results follow from the moment decomposition. In the first place the system closes on neglecting second and higher moments, a fact which lies at the heart of all known analytic reconnection solutions for incompressible plasmas-and this includes 3D reconnection models ${ }^{23,24}$ and solutions involving plane cylindrical coordinates with angular moments. ${ }^{25,26}$ This result also has echoes in the local expansion involved in the finite time collapse of a compressible $X$-point plasma. ${ }^{27}$

Second, if we proceed as below and limit attention to the "closed" moment system we find that the first order moments are autonomous, in the sense that they evolve independently of the behavior of the zeroth order fields. What this means is that the first order moments can be interpreted in terms of slowly evolving global fields: these "guide" or "background" fields control the transient, high frequency fields associated with the zero moments. This ordering is somewhat unusual, 
as in a typical expansion procedure we usually associate the zeroth-order terms with global background quantities and the first order terms with the small scale perturbations.

\section{The closed moment expansion}

By setting the second order and higher moments to zero and considering just the first two terms in the moment expansion we obtain for the zeroth moments

$$
\begin{aligned}
\phi_{x x t}^{0}+\left\langle\phi_{x x}^{0} \phi^{1}\right\rangle & =\left\langle\psi_{x x}^{0} \psi^{1}\right\rangle+\nu \phi_{x x x x}^{0}, \\
W_{t}^{0}+\left\langle W^{0} \phi^{1}\right\rangle= & \left\langle Z^{0} \psi^{1}\right\rangle+\nu W_{x x}^{0}, \\
\psi_{t}^{0}+\left\langle\psi^{0} \phi^{1}\right\rangle= & \eta \psi_{x x}^{0}+d_{i}\left\langle\psi^{0} Z^{1}\right\rangle+d_{e}^{2}\left(\psi_{x x t}^{0}+\left\langle\psi_{x x}^{0} \phi^{1}\right\rangle\right. \\
& \left.+\left\langle Z^{0} W^{1}\right\rangle\right), \\
Z_{t}^{0}+\left\langle Z^{0} \phi^{1}\right\rangle= & \eta Z_{x x}^{0}+\left\langle W^{0} \psi^{1}\right\rangle+d_{i}\left\langle\psi_{x x}^{0} \psi^{1}\right\rangle \\
& +d_{e}^{2}\left(Z_{x x t}^{0}+\left\langle Z_{x x}^{0} \phi^{1}\right\rangle+\left\langle\phi_{x x}^{0} Z^{1}\right\rangle\right) .
\end{aligned}
$$

Note that we have introduced brackets-remnants of the original Poisson brackets-typified by

$$
\left\langle f^{m} g^{n}\right\rangle=f_{x}^{m} g^{n}-f^{n} g_{x}^{m} .
$$

The first order moments are given by

$$
\begin{aligned}
\phi_{x x t}^{1}+\left\langle\phi_{x x}^{1} \phi^{1}\right\rangle & =\left\langle\psi_{x x}^{1} \psi^{1}\right\rangle+\nu \phi_{x x x x}^{1}, \\
W_{t}^{1}+\left\langle W^{1} \phi^{1}\right\rangle= & \left\langle Z^{1} \psi^{1}\right\rangle+\nu W_{x x}^{1}, \\
\psi_{t}^{1}+\left\langle\psi^{1} \phi^{1}\right\rangle= & \eta \psi_{x x}^{1}+d_{i}\left\langle\psi^{1} Z^{1}\right\rangle+d_{e}^{2}\left(\psi_{x x t}^{1}+\left\langle\psi_{x x}^{1} \phi^{1}\right\rangle\right. \\
& \left.+\left\langle Z^{1} W^{1}\right\rangle\right), \\
Z_{t}^{1}+\left\langle Z^{1} \phi^{1}\right\rangle= & \eta Z_{x x}^{1}+\left\langle W^{1} \psi^{1}\right\rangle+d_{i}\left\langle\psi_{x x}^{1} \psi^{1}\right\rangle \\
& +d_{e}^{2}\left(Z_{x x t}^{1}+\left\langle Z_{x x}^{1} \phi^{1}\right\rangle+\left\langle\phi_{x x}^{1} Z^{1}\right\rangle\right) .
\end{aligned}
$$

Of central interest are the allowable forms for the background field (remembering that these are given by the first order moments). In lieu of an exhaustive treatment we note that particular solutions can be constructed by observing that a bracket of the form

$$
\left\langle\phi_{x x}^{1} \phi^{1}\right\rangle=\phi_{x x x}^{1} \phi^{1}-\phi_{x x}^{1} \phi_{x}^{1}
$$

can be made to vanish by taking $\phi^{1}=\alpha x$ or alternatively $\phi^{1}$ $=\alpha(t) e^{i k x}$. The first form yields the background fields used in
Sec. III A, i.e., $\phi=\phi^{0}(x)+\phi^{1}(x) y=\phi^{0}(x)+\alpha x y$. The second form yields slowly evolving background fields, such as those outlined in Ref. 13. In this case if we assume similar forms for $W^{1}, \psi^{1}, Z^{1}$ and consider only odd solutions we find that

$$
\left(\begin{array}{c}
\phi^{1} \\
W^{1} \\
\psi^{1} \\
Z^{1}
\end{array}\right)=\sin (k x)\left(\begin{array}{c}
\alpha_{0} \exp \left(-\lambda_{1} t\right) \\
\gamma_{0} \exp \left(-\lambda_{1} t\right) \\
\beta_{0} \exp \left(-\lambda_{2} t\right) \\
\delta_{0} \exp \left(-\lambda_{2} t\right)
\end{array}\right),
$$

where $\lambda_{1}=\nu k^{2}$ and $\lambda_{2}=\eta k^{2} /\left(1+d_{e}^{2} k^{2}\right)$. If these solutions are to represent global background fields then the wavenumber $k$ should be taken to be of order unity. In this case the decay of the resulting guide field is negligible (because $\nu$ and $\eta$ are typically very small).

${ }^{1}$ E. R. Priest and T. G. Forbes, Magnetic Reconnection MHD Theory and Applications (Cambridge University Press, Cambridge, 2000).

${ }^{2}$ T. J. Schep, F. Pegoraro, and B. N. Kuvshinov, Phys. Plasmas 1, 2843 (1994).

${ }^{3}$ A. Bhattacharjee, Z. W. Ma, and X. Wang, J. Geophys. Res. 104, 14543 (1999).

${ }^{4}$ J. Birn, J. F. Drake, M. A. Shay, B. N. Rogers, R. E. Denton, M. Hesse, M. Kuznetsova, Z. W. Ma, A. Bhattacharjee, A. Otto et al., J. Geophys. Res. 106, 3715 (2001)

${ }^{5}$ M. A. Shay, J. F. Drake, B. N. Rogers, and R. E. Denton, J. Geophys. Res. 106, 3759 (2001).

${ }^{6}$ I. J. D. Craig and P. G. Watson, Sol. Phys. 214, 131 (2003).

${ }^{7}$ I. J. D. Craig, J. Heerikhuisen, and P. G. Watson, Phys. Plasmas 10, 3120 (2003).

${ }^{8}$ I. J. D. Craig and S. M. Henton, Astrophys. J. 450, 280 (1995).

${ }^{9}$ M. Jardine and H. R. Allen, Sol. Phys. 177, 411 (1998).

${ }^{10}$ J. C. Dorelli, Phys. Plasmas 10, 3309 (2003).

${ }^{11}$ M. A. Shay and J. F. Drake, Geophys. Res. Lett. 25, 3759 (1998).

${ }^{12}$ J. C. Dorelli and J. Birn, J. Geophys. Res. 108, 1133 (2003).

${ }^{13}$ I. J. D. Craig and P. G. Watson, Astrophys. J. 516, 924 (1999).

${ }^{14}$ I. J. D. Craig and P. G. Watson, Sol. Phys. 191, 359 (2000).

${ }^{15}$ I. J. D. Craig and P. G. Watson, Sol. Phys. 194, 251 (2000).

${ }^{16}$ R. Fitzpatrick, Phys. Plasmas 11, 937 (2004).

${ }^{17}$ R. Fitzpatrick, Phys. Plasmas 11, 3961 (2004).

${ }^{18}$ Y. E. Litvinenko and I. J. D. Craig, Sol. Phys. 189, 315 (1999).

${ }^{19}$ D. Biskamp and H. Welter, Phys. Rev. Lett. 44, 1069 (1980).

${ }^{20}$ G. J. Rickard and I. J. D. Craig, Phys. Fluids B 5, 956 (1993).

${ }^{21}$ P. G. Watson and F. Porcelli, "Exact steady-state reconnection solutions in weakly collisional plasmas," Astrophys. J. (to be published).

${ }^{22}$ A. Bhattacharjee, Z. W. Ma, and X. Wang, Phys. Plasmas 8, 1829 (2001).

${ }^{23}$ I. J. D. Craig, R. B. Fabling, S. M. Henton, and G. J. Rickard, Astrophys. J. Lett. 455, L197 (1995).

${ }^{24}$ I. J. D. Craig and R. B. Fabling, Astrophys. J. 462, 969 (1996).

${ }^{25}$ P. G. Watson and I. J. D. Craig, Sol. Phys. 207, 337 (2002).

${ }^{26}$ E. Tassi, V. S. Titov, and G. Hornig, Phys. Lett. A 315, 382 (2003).

${ }^{27}$ V. S. Imshennik and S. I. Syrovatskii, Sov. Phys. JETP 25, 656 (1967). 